\title{
Nonlinear Hysteretic Response of Piezoelectric Ceramics
}

\author{
Amir Sohrabi and Anastasia Muliana \\ Department of Mechanical Engineering \\ Texas AEM University, College Station, \\ USA
}

\section{Introduction}

Ferroelectric materials, such as lead zirconate titanate (PZT), have been widely used in sensor, actuator, and energy conversion devices. In this paper, we are primarily interested in the electro-mechanical response of polarized ferroelectric ceramics subject to cyclic electric fields at various magnitudes and frequencies. There have been experimental studies on understanding the effect of electric fields and loading rates on the overall electro-mechanical response of PZT (see for examples Crawley and Anderson 1990, Zhou and Kamlah 2006). The electrical and mechanical responses of PZT are also shown to be time-dependent, especially under high electric field (Fett and Thun 1998; Cao and Evans 1993; Schaufele and Hardtl, 1996). Ben Atitallah et al. (2010) studied the hysteretic response of PZT5A and active PZT fiber composite at several frequencies and isothermal temperatures. They show the nonlinear and time-dependent piezoelectric constants of the PZTs and PZT fiber composites. In a review of nonlinear response of piezoelectric ceramics, Hall (2001) discussed experimental studies that show strong time-dependent and nonlinear behavior in the electro-mechanical response of ferroelectric ceramics. The time-dependent effect becomes more prominent at electric fields close to the coercive electric field of the ferroelectric ceramics and under high magnitude of electric fields a ferroelectric ceramics exhibits nonlinear electro-mechanical response. Furthermore, high mechanical stresses could result in nonlinear mechanical, electrical, and electro-mechanical responses of the ferroelectric materials. Within a context of a purely mechanical loading in viscoelastic materials, timedependent response is shown by a stress relaxation (or a creep strain). This results in stressstrain hysteretic response when a viscoelastic material is subjected to a cyclic mechanical loading. There are different types of viscoelastic materials, such as polymers, biological tissues, asphalts, and geological materials. It is understood that these materials possess different microstructural characteristics at several length scales; however the macroscopic (overall) $^{1}$ mechanical response of these materials, i.e. stress relaxation and hysteretic response, especially for a linear response, follows similar trends. Experimental studies have shown that there are similarities with regards to the macroscopic time-dependent (or

\footnotetext{
1 In this context, by observing the macroscopic response of materials we treat the bodies as continuous and homogeneous with respect to their mechanical response although there is no clear cut as at which length scale the bodies can be considered continuous and homogeneous.
} 
frequency dependent) response of piezoelectric ceramics, i.e. electro-mechanical coupling, dielectric constant, and mechanical stress-strain relation, to the macroscopic time-dependent and hysteretic behaviors of viscoelastic materials although it is obvious that the microstructural morphologies of piezoelectric ceramics are completely different from the ones of viscoelastic materials mentioned above.

The macroscopic response of materials depends upon their microstructural changes when these materials are subjected to various histories of external stimuli such as mechanical load and/or electric field. Several constitutive models have been developed to examine the effect of electric field and mechanical stress on the overall hysteretic response of ferroelectric materials. These constitutive models can be classified as purely phenomenological models derived based on classical mechanics and thermodynamics framework and micromechanics based models that incorporate changes in the polycrystalline (micro) structure of ferroelectric ceramics with external stimuli. In the phenomenological models, changes in the microstructures of materials due to external stimuli are not explicitly modeled; however the effects of microstructural changes on the macroscopic response of materials can be incorporated by allowing the material parameters to vary with the external stimuli. It is also noted that any changes in micro- and macroscopic responses of materials occur in some finite period and in most cases these changes also depend upon how fast or slow the external stimuli are prescribed to the bodies, leading to what so called 'rate-dependent response'. Changes in the microstructures of materials with external stimuli are rather complicated. It might not be possible to incorporate detailed mechanisms that trigger these changes in developing constitutive models, mainly due to the complexity of these microstructural changes that occur at various scales and it is not well understood how the interactions among different field variables at the microscopic scale affects the macroscopic response. Several micromechanics based models are derived with a motivation to incorporate some aspects of the microstructural characteristics in predicting macroscopic response of materials. These micromechanics models are of course based on some assumptions and hypotheses. For examples: Smith et al. (2003, 2006) developed a constitutive model for hysteretic polarization switching response based on free-energy of a single crystal structure. It is assumed that the free energy is related to dipole reorientation in each crystal structure. A stochastic homogenization approach is used to obtain macroscopic hysteretic response of polycrystal structures. Chan and Hagood (1994) modeled a polarization reversal behavior of a single-crystal piezoceramic by assuming that the single crystal can be polarized to six possible directions and the overall responses of the piezoceramics are obtained either by averaging the crystallite responses in a global coordinate system or by taking into account internal alignment of the crystallites. Chen and Lynch (1998) incorporated the effects of grain orientations and crystal structures, i.e. tetragonal and rhombohedral, on the macroscopic hysteretic response of piezoelectric ceramics. These various microstructural-based models that require different material parameters are shown capable of simulating nonlinear hysteretic macroscopic response of piezoelectric ceramics. In most cases, these material parameters are characterized from the macroscopic response of the materials. An attempt of using molecular dynamics simulations in predicting macroscopic nonlinear hysteretic response of ferroelectric materials has also been considered. Such studies can be found in Uludogan et al. (2008) and Fang and Sang (2009). All of the above models that to some extents include microstructural aspects of piezoelectric ceramics are derived with a motivation to explain and improve understanding 
on the nonlinear hysteretic response; however it might be difficult if not possible to perform experiments that can trace detailed microstructural changes at various microscopic scales during the hysteretic response, not to mention incorporating the rate of these changes as well. A discussion on the development of constitutive models of ferroelectric materials can be found in Smith (2005) and Lines and Glass (2009).

Bassiouny et al. (1988a and b, 1989) formulated a phenomenological model for predicting electromechanical hysteretic response of piezoelectric ceramics. They defined a thermodynamic potential in terms of reversible and irreversible parts of the polarization. The irreversible part is the energy associated with the residual electric polarization. This constitutive model leads to rate-independent equations for the electro-mechanical coupling in piezoelectric ceramics (in analogy to the flow rule plasticity model). Huang and Tiersten (1998a and b) used a phenomenological based model for describing electro-mechanical hysteretic behavior in ferroelectric ceramics. Their model can capture the overall nonlinear hysteretic response, but it does not incorporate the effect of frequencies on the overall hysteretic response. Another example of phenomenological models of nonlinear rateindependent hysteretic response of piezoelectric ceramics is by Kamlah and Tsakmakis (1999). The nonlinearity is due to polarization switching when the piezoelectric ceramics are subjected to high electric field and compressive stress. Similar to the crystal plasticity model of Bassiouny et al. (1988a and b) Landis (2002) developed a phenomenological model for predicting polarization switching in ferroelectric materials. They used an idea of rateindependent plasticity model and discussed an extension of the constitutive model to include a rate-dependent response. Tiersten $(1971,1993)$ developed a nonlinear electroelastic model for predicting response of polarized piezoelectric ceramics undergoing large electric driving fields and small strains. The constitutive model includes higher order terms of electric fields. Crawley and Anderson (1990) suggested that the nonlinear electric field can be incorporated by taking a linear piezoelectric constant to depend on the electric field. Massalas et al. (1994) and Chen (2009) presented nonlinear thermo-electro-mechanical constitutive equations for elastic materials with memory-dependent (viz. viscoelastic materials) that incorporate the effect of heat generation due to the dissipation of energy on the nonlinear thermo-electro-mechanical response of conductive materials. The advantages of the phenomenological models are in their relatively simple forms in which the material parameters can be easily characterized from macroscopic experiments, which are beneficial for designing structures consisting of piezoelectric ceramics.

The electro-mechanical response of ferroelectric ceramics is shown to be time- (or rate-) dependent within a context of dielectric- and piezoelectric relaxation; however limited studies have been done on predicting time-dependent response of ferroelectric ceramics. We extend the concepts of response of viscoelastic solids to evaluate the nonlinear timedependent electro-mechanical (macroscopic) response of polarized ferroelectric materials, i.e. piezoelectric ceramics. General time-integral electro-mechanical phenomenological constitutive models based on multiple integral and nonlinear single integral forms are used. We assume that the dielectric and piezoelectric constants of the materials change with electric field and the rate of time-dependent polarization and strain responses can also change with the magnitude of the electric field. This manuscript is organized as follows. Section two discusses a nonlinear time-dependent constitutive model based on integral formulations for electro-mechanical response of piezoelectric ceramics, followed by numerical implementation and verification of the models in section three. Section four 
presents analyses of piezoelectric bimorph actuators having time-dependent material properties. The last section is dedicated to a conclusion and a discussion of the proposed nonlinear time-integral models.

\section{Nonlinear time-dependent constitutive model for piezoelectric ceramics}

\subsection{Nonlinear electro-elastic constitutive model}

A phenomenological constitutive model $^{2}$ for polarized ferroelectric ceramics at an isothermal condition is described in terms of the following field variables: stress $\sigma$, strain $\varepsilon$, electric field E, electric flux (displacement) $\mathbf{D}$. It is assumed that loading is within a quasistatic condition such that the effect of inertia on the electro-mechanical response can be neglected. The constitutive model for polarized ferroelectric ceramics can be obtained by defining a thermodynamic potential $\psi_{e}(\boldsymbol{\varepsilon}, \mathbf{E})$ (see Bassiouny et al. 1988a; Tiersten, 1993; Huang and Tiersten, 1998). The relations between the different field variables are obtained from:

$$
\sigma_{i j}=\left.\frac{\partial \psi_{e}}{\partial \varepsilon_{i j}}\right|_{\mathrm{E}} \quad D_{i}=-\left.\frac{\partial \psi_{e}}{\partial E_{i}}\right|_{\sigma}
$$

The components of the electric field and strain are expresses as $E_{i}=-\varphi_{, i}$ and $\varepsilon_{i j}=\frac{1}{2}\left(u_{i, j}+u_{j, i}\right)$, respectively; where $\varphi$ and $u_{i}$ are the electric potential and scalar component of the displacement, respectively. This study focuses on understanding response of piezoelectric ceramics undergoing large electric fields and the brittle nature of piezoelectric ceramics limits their deformation to small strains. The thermodynamic potential includes up to second order strain tensor and higher order electric field. Tiersten (1993) suggested the following free energy function at an isothermal condition:

$$
\psi_{e}=\frac{1}{2} C_{i j k l} \varepsilon_{i j} \varepsilon_{k l}-e_{i j k} \varepsilon_{j k} E_{i}-\frac{1}{2} \kappa_{i j} E_{i} E_{j}-\frac{1}{2} b_{i j k l} E_{i} E_{j} \varepsilon_{k l}-\frac{1}{6} \chi_{i j k} E_{i} E_{j} E_{k}+H . O . T
$$

where $C_{i j k l}, e_{i j k}, \kappa_{i j}, b_{i j k l}$, and $\chi_{i j k}$ are the fourth-order elasticity tensor, third-order electromechanical tensor (piezoelectric constant), second-order electric permeability (dielectric constant), fourth-order electro-mechanical tensor, and third-order electric permeability tensor, respectively. The above elasticity constants are measured at constant or zero electric field, while the electrical properties are measured at constant or zero strains. The stress and electric displacement are:

$$
\begin{aligned}
& \sigma_{i j}=C_{i j k l} \varepsilon_{k l}-e_{k i j} E_{k}-\frac{1}{2} \hat{b}_{k i j} E_{k} E_{l} \\
& D_{i}=e_{i j k} \varepsilon_{j k}+\hat{\kappa}_{i j} E_{j}+\frac{1}{2} \chi_{i j k} E_{j} E_{k}
\end{aligned}
$$

\footnotetext{
2 We deal with a constitutive model for a continuous and homogeneous body, suitable for simulating response of a piezoelectric ceramic below its coercive electric field.
} 
where

$$
\begin{aligned}
& \hat{b}_{i j k l}=b_{i j k l}-2 \kappa_{o}\left(\delta_{k i} \delta_{l j}-\frac{1}{2} \delta_{i j} \delta_{k l}\right) \\
& \hat{\kappa}_{i j}=\kappa_{i j}^{\varepsilon}=\kappa_{o} \delta_{i j}+\kappa_{i j}
\end{aligned}
$$

Here $\kappa_{o}$ is the permittivity constant at free space and $\delta_{i j}$ is the delta Kronecker. Tiersten (1993) also discussed an alternative expression of the constitutive model with nonlinear electric field and small strain when stress, electric field, and temperature are taken as the independent field variables:

$$
\begin{aligned}
& \varepsilon_{i j}=S_{i j k l} \sigma_{k l}+d_{k i j} E_{k}+\frac{1}{2} f_{k l i j} E_{k} E_{l} \\
& D_{i}=d_{i j k} \sigma_{j k}+\kappa_{i k}^{\sigma} E_{k}+\frac{1}{2} \chi_{i j k}^{\sigma} E_{j} E_{k}
\end{aligned}
$$

The elastic compliances, $S_{i j k l}$, piezoelectric constant, $d_{i j k}$, and nonlinear electroelastic constants, $f_{i j k l}$, are:

$$
\begin{aligned}
S_{i j k l} & =C_{i j k l}^{-1} \\
d_{i j k} & =e_{i m n} S_{m n j k} \\
f_{i j k l} & =\hat{b}_{i j m n} S_{m n k l}
\end{aligned}
$$

The second- and third-order electric permeability constants are measured at zero or constant stresses:

$$
\begin{aligned}
& \kappa_{i j}^{\sigma}=\hat{\kappa}_{i j}+e_{i m n} d_{j m n} \\
& \chi_{i j k}^{\sigma}=\chi_{i j k}+e_{i m n} f_{j k m n}
\end{aligned}
$$

\subsection{Nonlinear time-dependent constitutive model}

In analogy to the time-dependent deformation of viscoelastic materials, we extend the nonlinear electro-elastic model developed by Tiersten (1993) to include time-dependent material parameters. There have been several integral models developed to describe nonlinear viscoelastic behavior: modified superposition principle (Findley and Lai, 1967), multiple integral model (Green and Rivlin 1957), finite strain integral models (Pipkins and Rogers 1968; Rajagopal and Wineman 2010), single integral models (Pipkins and Rogers 1968; Schapery 1969), and quasi-linear viscoelastic model (Fung 1981). The work by Green and Rivlin (1957) provides the fundamental framework for nonlinear viscoelastic response using the principles of continuum mechanics. It is assumed that small changes in the input field variables cause only small changes in the corresponding output field variables; this can be approximated by using continuous functions by polynomials. For a nonlinear viscoelastic material, Green and Rivlin (1957) formed a sum of multiple integrals of the polynomial functions to incorporate history of input variables in predicting output at current time. The constitutive equations (2.3) and (2.5) are expressed in the polynomial functions of independent field variables. In analogy to the correspondence between elastic and viscoelastic materials, we extend the nonlinear electro-elastic equations of Tiersten (1993) to include the time-dependent effect (for non-aging materials): 


$$
\begin{aligned}
& \varepsilon_{i j}(t)=\int_{0^{-}}^{t} S_{i j k l}(t-s) \frac{d \sigma_{k l}(s)}{d s} d s+\int_{0^{-}}^{t} d_{k j j}(t-s) \frac{d E_{k}(s)}{d s} d s+\frac{1}{2} \int_{0^{-}}^{t} \int_{0^{-}}^{t} f_{k l i j}\left(t-s_{1}, t-s_{2}\right) \frac{d E_{k}\left(s_{1}\right)}{d s_{1}} \frac{d E_{l}\left(s_{2}\right)}{d s_{2}} d s_{1} d s_{2} \\
& D_{i}(t)=\int_{0^{-}}^{t} d_{i j k}(t-2) \frac{d \sigma_{j k}(s)}{d s} d s+\int_{0^{-}}^{t} \kappa_{i k}^{\sigma}(t-s) \frac{d E_{k}(s)}{d s} d s+\frac{1}{2} \int_{0^{-}}^{t} \int_{0^{-}}^{t} \chi_{i j k}^{\sigma}\left(t-s_{1}, t-s_{2}\right) \frac{d E_{j}\left(s_{1}\right)}{d s_{1}} \frac{d E_{k}\left(s_{2}\right)}{d s_{2}} d s_{1} d s_{2}
\end{aligned}
$$

It is also possible to include higher order terms of the electric field. In order to graphically visualize the linear and nonlinear kernel functions of time, let us consider a one-dimensional multiple integral forms (up to the second order):

$$
R(t)=\int_{0^{-}}^{t} \varphi_{1}(t-s) \frac{d I(s)}{d s} d s+\int_{0^{-}}^{t} \int_{0^{-}}^{t} \varphi_{2}\left(t-s_{1}, t-s_{2}\right) \frac{d I\left(s_{1}\right)}{d s_{1}} \frac{d I\left(s_{2}\right)}{d s_{2}} d s_{1} d s_{2}
$$

where $R(t)$ is the corresponding output at current time $t, I(s)$ is the input history prescribed at $0^{-} \leq s \leq t, \varphi_{1}(t)$ and $\varphi_{2}(t, t)$ are the two kernel functions. When the kernels are assumed to increase with time, Fig. 2.1 illustrates the linear and second order kernel functions of time (see Findley et al. (1976) for a detailed explanation). It is also assumed that the material response is unaltered by an arbitrary shift of the time scale, so that $\varphi_{2}\left(t, t-s_{1}\right)=\varphi_{2}\left(t-s_{1}, t\right)$. The following functions can be used for the two kernels in Eq. (2.9):

$$
\begin{aligned}
& \varphi_{1}(t-s)=A_{0}+A_{1}\left(1 .-e^{-(t-s) / \tau_{1}}\right) \\
& \varphi_{2}\left(t-s_{1}, t-s_{2}\right)=B_{0}+B_{1}\left(2 .-e^{-\left(t-s_{1}\right) / \lambda_{1}}-e^{-\left(t-s_{2}\right) / \lambda_{1}}\right)+B_{2}\left(1 .-e^{-\left(t-s_{1}\right) / \lambda_{2}}\right)\left(1 .-e^{-\left(t-s_{2}\right) / \lambda_{2}}\right)
\end{aligned}
$$

where $A_{0}, A_{1}, B_{0}, B_{1}, B_{2}, \tau_{1}, \lambda_{1}, \lambda_{2}$ are the material parameters that need to be determined from experiments. A set of experiments may be performed by applying the input variables at different times, say at $t=0$ and $t=\mathrm{s}_{1}$. The main disadvantage of the multiple integral forms is in characterizing material parameters from experiments, even when only up to the second order kernel function is considered. The characterization of material parameters becomes even more complicated for the anisotropic and nonlinear time-dependent case, which is the case for piezoelectric ceramics.
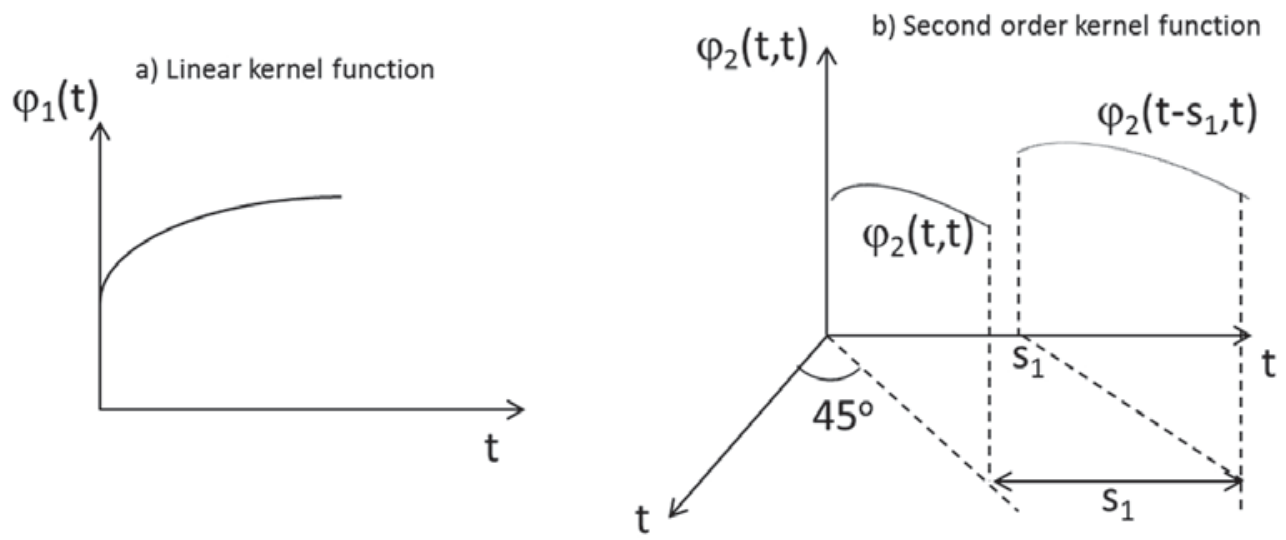

Fig. 2.1. Time-dependent kernel functions (see Findley et al., 1976) 
It is also possible to include higher order terms of the electric field. In case of the third order term is included, the following third order kernel function can be considered:

$$
\begin{aligned}
& \varphi_{3}\left(t-s_{1}, t-s_{2}, t-s_{3}\right)=C_{0}+C_{1}\left(3 .-e^{-\left(t-s_{1}\right) / \eta_{1}}-e^{-\left(t-s_{2}\right) / m_{1}}-e^{-\left(t-s_{3}\right) / \eta_{1}}\right)+ \\
& C_{2}\left(1 .-e^{-\left(t-s_{1}\right) / \eta_{2}}\right)\left(1 .-e^{-\left(t-s_{2}\right) / \eta_{2}}\right)\left(1 .-e^{-\left(t-s_{3}\right) / \eta_{2}}\right)
\end{aligned}
$$

It is also necessary that $\varphi_{3}\left(t, t, t-s_{1}\right)=\varphi_{3}\left(t-s_{1}, t, t\right)=\varphi_{3}\left(t, t-s_{1}, t\right)$.

To reduce complexity in analyzing nonlinear viscoelastic behavior and characterizing material properties a single integral with nonlinear integrand has been used and found capable of approximating nonlinear responses in viscoelastic materials. Such models are discussed in Findley and Lay (1966), Pipkins and Rogers (1968), and Schapery (1969). Chen (2009) derived a nonlinear thermo-electro-viscoelastic constitutive equation that incorporates heat generation due to the dissipation of energy ${ }^{3}$ and damage. The Gibbs free energy is defined in terms of a functional of the histories of stress, temperature, temperature gradient and electric field in the reference configuration and damage is introduced as an internal state variable. This constitutive model is based on a single integral form that includes hysteresis, aging, and damage in the electro-active materials, written as:

$$
\begin{aligned}
\frac{1}{2} C_{i j}(t)= & L_{i j}^{0}+\int_{-\infty}^{t} J_{i j k l}(0, t-s, \mathbf{d}) \frac{d \Sigma_{k l}(s)}{d s} d s+\int_{-\infty}^{t} \alpha_{i j}(0, t-s, \mathbf{d}) \frac{d T(s)}{d s} d s+ \\
& \int_{-\infty}^{t} f_{i j k}(0, t-s, \mathbf{d}) \frac{d E_{k}(s)}{d s} d s \\
\rho_{o} s(t)= & M^{0}+\int_{-\infty}^{t} \alpha_{i j}(t-x, 0, \mathbf{d}) \frac{d \Sigma_{i j}(x)}{d x} d x+\frac{1}{T_{0}} \int_{-\infty}^{t} C_{g}(t-x, 0, \mathbf{d}) \frac{d T(x)}{d x} d x+ \\
& \int_{-\infty}^{t} \eta_{i}(t-x, 0, \mathbf{d}) \frac{d E_{i}(x)}{d x} d x \\
D_{i}(t)= & N_{i}^{0}+\int_{-\infty}^{t} f_{i j k}(t-x, 0, \mathbf{d}) \frac{d \Sigma_{j k}(x)}{d x} d x+\int_{-\infty}^{t} \eta_{i}(0, t-s, \mathbf{d}) \frac{d T(s)}{d s} d s+ \\
& \int_{-\infty}^{t} \kappa_{i j}(0, t-s, \mathbf{d}) \frac{d E_{j}(s)}{d s} d s
\end{aligned}
$$

where $\mathbf{C}, \boldsymbol{\Sigma}, \mathbf{D}, \mathbf{E}, T$, s are the right Cauchy-Green stretch tensor, second Piola-Kirchoff stress tensor, electric displacement vector, electric field vector, temperature, and entropy, respectively; $\rho_{0}$ is the mass density at a reference state; $\mathbf{L}^{0}, \mathbf{M}^{0}, \mathbf{N}$ are the right Cauchy-Green stretch tensor, product of the entropy and mass density, and electric displacement tensor at a reference state; $\mathbf{J}, \mathbf{a}, \mathbf{f}, \mathbf{n}, \mathbf{K}, \mathrm{C}_{8}$ are the compliance, thermal expansion, piezoelectric constant, pyroelectric constant, dielectric, and heat capacity, respectively; $T_{0}$ is the reference temperature; and $\mathbf{d}$ is the damage tensor. It is also necessary that each kernel in Eq. (2.12) satisfy the following condition: $\varphi(t-x, t-s, \mathbf{d})=\varphi(t-s, t-x, \mathbf{d})$. Chen (2009) also discussed

\footnotetext{
${ }^{3}$ Viscoelastic materials are known to dissipate significant amount of energy during cyclic loading; an electric current flows through a piezoelectric materials also dissipate energy which is converted to heat. Thus, it is necessary to account for this heat generation in predicting time-dependent response of piezoelectric materials.
} 
the time-dependent forms for each material property in Eq. (2.12) in order to incorporate aging and damage.

If we follow an approach suggested by Crawley and Anderson (1990) in which the nonlinear electric field can be incorporated by taking a linear piezoelectric constant to depend on the electric field, a single integral model with nonlinear integrand as the first approximation for modeling the time-dependent electro-mechanical response with nonlinearity due to high electric field is expressed ${ }^{4}$ as:

$$
\begin{aligned}
& \varepsilon_{i j}(t)=\int_{0^{-}}^{t} S_{i j k l}(t-s) \frac{d \sigma_{k l}(s)}{d s} d s+\int_{0^{-}}^{t} \frac{\partial R_{i j}}{\partial E_{k}}(t-s) \frac{d E_{k}(s)}{d s} d s \\
& D_{i}(t)=\int_{0^{-}}^{t} d_{i j k}(t-s) \frac{d \sigma_{j k}(s)}{d s} d s+\int_{0^{-}}^{t} \frac{\partial F_{i}}{\partial E_{k}}(t-s) \frac{d E_{k}(s)}{d s} d s
\end{aligned}
$$

where $R_{i j}\left[E_{k}(t-s), t\right]$ and $F_{i}\left[E_{k}(t-s), t\right]$ are the scalar components of the time-dependent strain and electric displacement, respectively, at current time $t \geq 0$ due to an input history of $E_{k}(s)$. It is assumed that $R_{i j}[0, t]=F_{i}[0, t]=0$ and $R_{i j}\left[E_{k}(t), t\right]=F_{i}\left[E_{k}(t), t\right]=0 \quad \forall t<0.0$. The following kernels can be used for the material parameters in the constitutive models in Eq. (2.13):

$$
\begin{aligned}
& S_{i j k l}(t)=S_{i j k l}^{(0)}+S_{i j k l}^{(1)}\left[1 .-e^{\left.-t / \tau_{i j k l}^{(1)}\right]}\right. \\
& d_{i j k}(t)=d_{i j k}^{(0)}+d_{i j k}^{(1)}\left[1 .-e^{-t / \tau} d_{i j k}^{(1)}\right] \\
& R_{i j}\left(E_{k}(0), t\right)=R_{i j}^{(0)}\left(E_{k}(0)\right)+R_{i j}^{(1)}\left(E_{k}(0)\right)\left[1 .-e^{-t / \tau^{(1)}} R_{i j}\right] \\
& F_{i}\left(E_{k}(0), t\right)=F_{i}^{(0)}\left(E_{k}(0)\right)+F_{i}^{(1)}\left(E_{k}(0)\right)\left[1 .-e^{-t / \tau_{F}^{(1)}}\right]
\end{aligned}
$$

It can be seen that the above kernels reduce to time-independent functions by eliminating the second term from the material parameters. By choosing $R_{i j}^{(0)}\left(E_{k}(0)\right), R_{i j}^{(1)}\left(E_{k}(0)\right), F_{i}^{(0)}\left(E_{k}(0)\right), F_{i}^{(1)}\left(E_{k}(0)\right)$ to vary linearly with the electric field, the above equation reduces to a linear time-dependent electro-mechanical coupling model. It is also possible to include more than one term for the time-dependent parts in Eq. (2.14). The timedependent compliance $S_{i j k l}(t)$ and piezoelectric constants $d_{i j k}(t)$ in Eq. (2.14) can be characterized from creep test by applying constant stresses or from hysteretic response due to cyclic stress inputs at different frequencies. The components of strain $R_{i j}(t)$ and electric displacement $F_{i}(t)$ can be determined from the hysteretic response due to sinusoidal electric field inputs at different amplitudes and frequencies. If the experimental setup permits for

4 This approach yields to a nonlinear single integral model of Pipkins and Rogers (1968). 
applying a fixed electric field, then the time-dependent strain and electric displacement can also be determined from this test. It is noted that for a piezoelectric ceramic such as a polarized PZT (let $x_{3}$ be the poling axis), only some of the components of the material parameters are nonzero, reducing the experimental effort in calibrating these parameters.

\subsection{Time-integration methods}

We present a numerical algorithm for determining time-dependent response of strain and electric displacement due to arbitrary stress and electric field inputs. We start with a numerical algorithm for one-dimensional single integral model with a nonlinear integrand and followed by an algorithm for multiple integral representations.

Let $R[I(t-s), t]$ be the time-dependent response at current time $t \geq 0$ due to an input history $I^{s} \equiv I(s)$. A general single integral representation for the response is:

$$
R^{t} \equiv R\left[I^{s}, t\right]=R\left[I^{0}, t\right]+\int_{0+}^{t} \frac{\partial R}{\partial I}\left[I^{s}, t-s\right] \frac{d I^{s}}{d s} d s \quad t \geq 0
$$

where

$$
\begin{gathered}
R\left[I^{0}, t\right]=R_{0}\left(I^{0}\right)+R_{1}\left(I^{0}\right)\left(1 .-\exp \left[-\frac{t}{\tau_{1}}\right]\right) \\
\frac{\partial R}{\partial I}\left[I^{s}, t-s\right]=\frac{\partial R_{0}\left(I^{s}\right)}{\partial I}+\frac{\partial R_{1}\left(I^{s}\right)}{\partial I}\left(1 .-\exp \left[-\frac{t-s}{\tau_{1}}\right]\right)
\end{gathered}
$$

Here we use a superscript to denote the time-dependent variables. A recursive method is used for solving the above integral form. Substituting Eqs. (2.16) and (2.17) into Eq. (2.15) yields:

$$
R^{t}=R_{0}\left(I^{t}\right)+R_{1}\left(I^{t}\right)-R_{1}\left(I^{0}\right) \exp \left[-\frac{t}{\tau_{1}}\right]-q^{t}
$$

where

$$
q^{t}=\int_{0^{+}}^{t} \frac{\partial R_{1}\left(I^{s}\right)}{\partial I} \exp \left[-\frac{t-s}{\tau_{1}}\right] \frac{d I^{s}}{d s} d s
$$

is the history variable, which can be approximated as:

$$
q^{t} \approx \exp \left[-\frac{\Delta t}{\tau_{1}}\right] q^{t-\Delta t}+\left[\left.\frac{\partial R_{1}\left(I^{t}\right)}{\partial I} \frac{d I}{d s}\right|_{t}+\left.\exp \left[-\frac{\Delta t}{\tau_{1}}\right] \frac{\partial R_{1}\left(I^{t-\Delta t}\right)}{\partial I} \frac{d I}{d s}\right|_{t-\Delta t}\right] \frac{\Delta t}{2} \quad t>0.0
$$

The superscript $t-\Delta t$ denotes the previous time history. At initial time, $q^{t}=q^{0}=0.0$ and $R^{0}=R_{0}\left(I^{0}\right)$. Equations (2.18) and (2.19) give the corresponding output due to an arbitrary input $I(s)$. For the multi-axial constitutive relation, the approximate solution in Eq. (2.18) can be applied independently to each scalar component in Eq. (2.13). 
The numerical algorithm for the multiple integral models (one-dimensional representation) in Eq. (2.9) with the kernels defined in Eq. (2.10) can be approximated by applying the recursive method as discussed above. The linear kernel is approximated as:

$$
\begin{gathered}
\int_{0^{-}}^{t} \varphi_{1}(t-s) \frac{d I(s)}{d s} d s \approx\left[A_{0}+A_{1}\right] I(t)-A_{1} I\left(0^{+}\right) \exp \left[-\frac{t}{\tau_{1}}\right]-q_{1}^{t} \\
q_{1}^{t} \approx \exp \left[-\frac{\Delta t}{\tau_{1}}\right] q_{1}^{t-\Delta t}+A_{1} \frac{\Delta t}{2}\left[\left.\frac{d I}{d s}\right|_{t}+\left.\exp \left[-\frac{\Delta t}{\tau_{1}}\right] \frac{d I}{d s}\right|_{t-\Delta t}\right] \quad t>0.0
\end{gathered}
$$

The second order kernel is rewritten as:

$$
\begin{aligned}
& \int_{0^{-}}^{t} \int_{0^{-}}^{t} \varphi_{2}\left(t-s_{1}, t-s_{2}\right) \frac{d I\left(s_{1}\right)}{d s_{1}} \frac{d I\left(s_{2}\right)}{d s_{2}} d s_{1} d s_{2}= \\
& {\left[B_{0}+B_{1}\left(2 .-e^{-t / \lambda_{1}}-e^{-t / \lambda_{1}}\right)+B_{2}\left(1 .-e^{-t / \lambda_{2}}\right)\left(1 .-e^{-t / \lambda_{2}}\right)\right] I\left(0^{+}\right) I\left(0^{+}\right)+} \\
& \int_{0^{+}}^{t} \int_{0^{+}}^{t}\left[B_{0}+B_{1}\left(2 .-e^{-\left(t-s_{1}\right) / \lambda_{1}}-e^{-\left(t-s_{2}\right) / \lambda_{1}}\right)+B_{2}\left(1 .-e^{-\left(t-s_{1}\right) / \lambda_{2}}\right)\left(1 .-e^{-\left(t-s_{2}\right) / \lambda_{2}}\right)\right] \frac{d I\left(s_{1}\right)}{d s_{1}} \frac{d I\left(s_{2}\right)}{d s_{2}} d s_{1} d s_{2}
\end{aligned}
$$

and it can be approximated by:

$$
\begin{aligned}
& \int_{0^{-}}^{t} \int_{0^{-}}^{t} \varphi_{2}\left(t-s_{1}, t-s_{2}\right) \frac{d I\left(s_{1}\right)}{d s_{1}} \frac{d I\left(s_{2}\right)}{d s_{2}} d s_{1} d s_{2} \approx \\
& {\left[B_{0}+B_{1}\left(2 .-e^{-t / \lambda_{1}}-e^{-t / \lambda_{1}}\right)+B_{2}\left(1 .-e^{-t / \lambda_{2}}\right)\left(1 .-e^{-t / \lambda_{2}}\right)\right] I\left(0^{+}\right) I\left(0^{+}\right)+} \\
& \left(B_{0}+2 B_{1}\right)\left(I(t)-I\left(0^{+}\right)\right)^{2}-B_{1}\left(I(t)-I\left(0^{+}\right)\right)\left(f_{1}^{t}+g_{1}^{t}\right)+ \\
& B_{2}\left[\left(I(t)-I\left(0^{+}\right)\right)^{2}-f_{2}^{t}\left(I(t)-I\left(0^{+}\right)\right)-g_{2}^{t}\left(I(t)-I\left(0^{+}\right)\right)+f_{2}^{t} g_{2}^{t}\right]
\end{aligned}
$$

where the history variables $f_{1}^{t}, f_{2}^{t}, g_{1}^{t}, g_{2}^{t}$ at $t>0.0$ are given as:

$$
\begin{aligned}
& f_{1}^{t} \approx \exp \left[-\frac{\Delta t}{\lambda_{1}}\right] f_{1}^{t-\Delta t}+\frac{\Delta t}{2}\left[\left.\frac{d I}{d s_{1}}\right|_{t}+\left.\exp \left[-\frac{\Delta t}{\lambda_{1}}\right] \frac{d I}{d s_{1}}\right|_{t-\Delta t}\right] \\
& g_{1}^{t} \approx \exp \left[-\frac{\Delta t}{\lambda_{1}}\right] g_{1}^{t-\Delta t}+\frac{\Delta t}{2}\left[\left.\frac{d I}{d s_{2}}\right|_{t}+\left.\exp \left[-\frac{\Delta t}{\lambda_{1}}\right] \frac{d I}{d s_{2}}\right|_{t-\Delta t}\right] \\
& f_{2}^{t} \approx \exp \left[-\frac{\Delta t}{\lambda_{2}}\right] f_{2}^{t-\Delta t}+\frac{\Delta t}{2}\left[\left.\frac{d I}{d s_{1}}\right|_{t}+\left.\exp \left[-\frac{\Delta t}{\lambda_{2}}\right] \frac{d I}{d s_{1}}\right|_{t-\Delta t}\right] \\
& g_{2}^{t} \approx \exp \left[-\frac{\Delta t}{\lambda_{2}}\right] g_{2}^{t-\Delta t}+\frac{\Delta t}{2}\left[\left.\frac{d I}{d s_{2}}\right|_{t}+\left.\exp \left[-\frac{\Delta t}{\lambda_{2}}\right] \frac{d I}{d s_{2}}\right|_{t-\Delta t}\right]
\end{aligned}
$$

At initial time, $f_{1}^{0}=f_{2}^{0}=g_{1}^{0}=g_{2}^{0}=0.0$ and $R(0)=A_{0} I\left(0^{+}\right)+B_{0} I\left(0^{+}\right) I\left(0^{+}\right)$. Thus, the corresponding response due to an arbitrary input obtained from the multiple integral model is approximated as: 


$$
\begin{aligned}
& R(t) \approx\left[A_{0}+A_{1}\right] I(t)-A_{1} \exp \left[-\frac{t}{\tau_{1}}\right]-q_{1}^{t}+ \\
& {\left[B_{0}+B_{1}\left(2 .-e^{-t / \lambda_{1}}-e^{-t / \lambda_{1}}\right)+B_{2}\left(1 .-e^{-t / \lambda_{2}}\right)\left(1 .-e^{-t / \lambda_{2}}\right)\right] I\left(0^{+}\right) I\left(0^{+}\right)+} \\
& \left(B_{0}+2 B_{1}\right)\left(I(t)-I\left(0^{+}\right)\right)^{2}-B_{1}\left(I(t)-I\left(0^{+}\right)\right)\left(f_{1}^{t}+g_{1}^{t}\right)+ \\
& B_{2}\left[\left(I(t)-I\left(0^{+}\right)\right)^{2}-f_{2}^{t}\left(I(t)-I\left(0^{+}\right)\right)-g_{2}^{t}\left(I(t)-I\left(0^{+}\right)\right)+f_{2}^{t} g_{2}^{t}\right]
\end{aligned}
$$

For the multi-axial constitutive relation, the approximate solution in Eq. (2.26) can be applied independently to each scalar component in Eq. (2.8).

\section{Numerical implementation and parametricstudies}

We present a numerical implementation of the above time-dependent constitutive models. We include parametric studies on understanding the effects of different material parameters and input histories on the overall time-dependent response of polarized ferroelectric materials. Both nonlinear single integral and multiple integral models will be discussed.

\subsection{Single integral model}

This section deals with using a single integral model to simulate hysteretic response of a polarized ferroelectric, which focuses on PZTs, subject to a sinusoidal electric field input. Let $x_{3}$ be the poling axis of the PZT and an electric field input $E_{3}(s)=E_{\max } \sin \omega t$ is applied along the poling axis. We consider several case studies: linear time-dependent response at a stress free condition, nonlinear time-dependent response at a stress free condition, and response under a combine mechanical stress and electric field. The following material parameters are considered for the linear time-dependent electro-mechanical coupling and dielectric constant:

$$
\begin{aligned}
& d_{333}(t)=\left[380+150\left(1 .-e^{-t / 5}\right)\right] \cdot 10^{-12} \mathrm{C} / N(\mathrm{~m} / \mathrm{V}) \\
& d_{311}(t)=d_{322}(t)=\left[-200-100\left(1 .-e^{-t / 2}\right)\right] \cdot 10^{-12} \mathrm{C} / \mathrm{N}(\mathrm{m} / \mathrm{V}) \\
& d_{113}=d_{223}=437 \cdot 10^{-12} \mathrm{C} / \mathrm{N}(\mathrm{m} / \mathrm{V}) \\
& \kappa_{33}^{\sigma}(t)=\left[23+2.3 e^{-t / 10}\right] \cdot 10^{-9} \mathrm{~F} / \mathrm{m} \\
& \kappa_{11}^{\sigma}=\kappa_{22}^{\sigma}=30 \cdot 10^{-9} \mathrm{~F} / \mathrm{m}
\end{aligned}
$$

The first case considers sinusoidal electric field inputs at three different frequencies: 0.01, 0.1 , and $1 \mathrm{~Hz}$ and two amplitudes: 0.25 and $0.75 \mathrm{MV} / \mathrm{m}$. Figure 3.1 illustrates the electricfield and transverse strain $\left(E_{3}-\varepsilon_{11}\right)$ response during the first quarter cycle. It is seen that the electric-field and strain curves show nonlinear behavior which is due to the delay (timedependent) response of the material. The nonlinearity is more pronounced as the frequency decreases. At the frequency $1 \mathrm{~Hz}$ the curve shows almost a linear behavior as the electric field is applied relatively fast with regards to the characteristics time of the materials and thus only a little time is given for the material to experience a time-dependent (or relaxation) effect. From Eq. (3.1), the characteristics time of the electro-mechanical coupling $d_{311}$ is 2 seconds. Thus, one should be very careful when interpreting an experimental data that involves nonlinear phenomena. As shown in Fig. 3.1, the response seems to suggest the 
nonlinear relation between the electric field and transverse strain which can be attributed to the electric field (or strain) dependent material properties, but instead this nonlinearity is due to the linear time-dependent effect. Figure 3.2 shows a hysteretic response of a PZT material at frequency $0.1 \mathrm{~Hz}$ and maximum applied electric field of $0.25 \mathrm{MV} / \mathrm{m}$. Experimental data are obtained from Crawley and Anderson (1990). The single integral model with time-dependent material parameter in Eq. (3.1) is shown to be capable of simulating the hysteretic response.
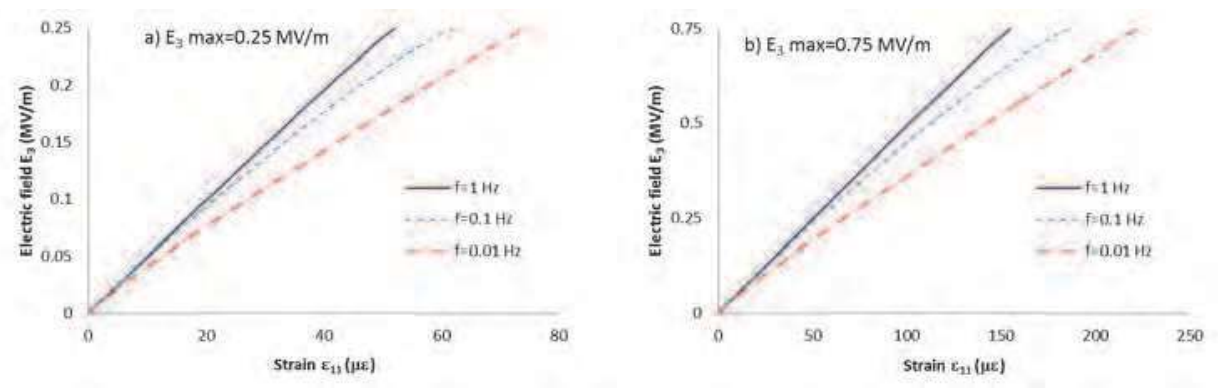

Fig. 3.1. Transverse strain responses during the first quarter cycle of the sinusoidal input

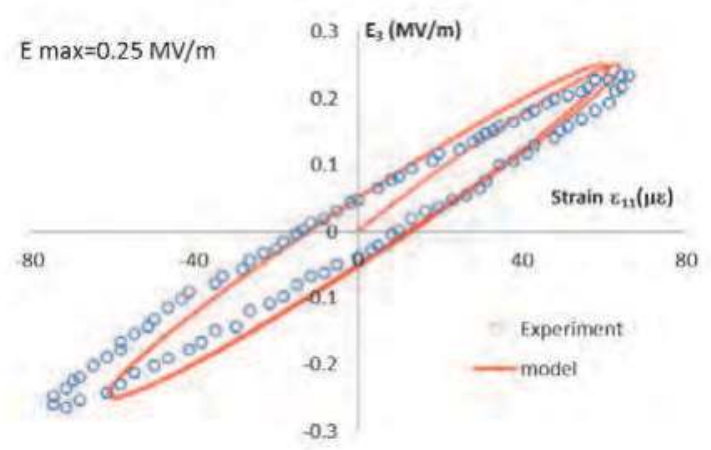

Fig. 3.2. Linear hysteretic response of a PZT at $\mathrm{f}=0.1 \mathrm{~Hz}$
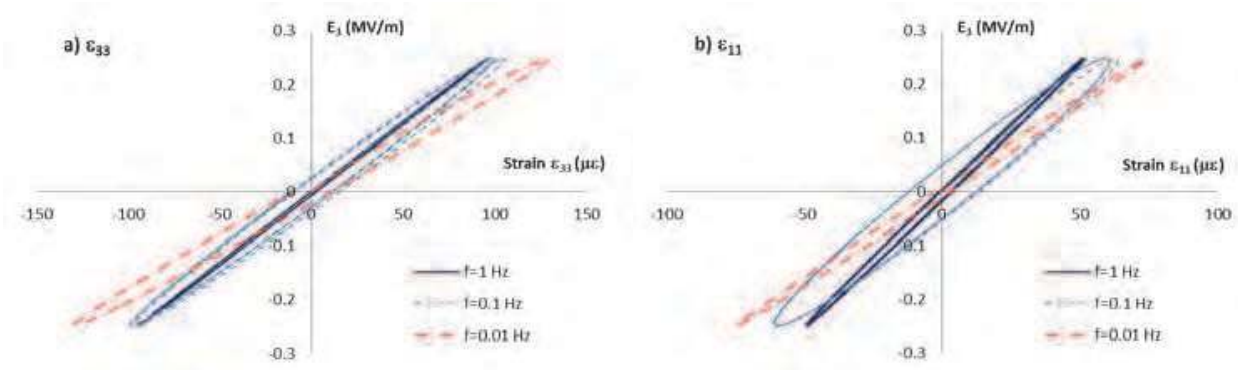

Fig. 3.3. Linear hysteretic response of the axial and transverse strains 
The effect of frequencies on the hysteretic response of the linear single integral model is illustrated in Fig. 3.3. It is noted that the strain along the poling axis is a compressive strain since the electric field is applied opposite to the poling direction to create elongation in the in-plane (transverse) direction. From Eq. (3.1) it can be seen that the characteristics time in $d_{311}$ is smaller than the one in $d_{333}$; thus, the transverse strain exhibits faster relaxation when subjected to an electric field along the poling axis. When the rate of loading is comparable to the characteristics time, the effect of time-dependent material properties on the hysteretic response becomes significant, as shown by the response with a frequency of $0.1 \mathrm{~Hz}$. When the rate of loading is relatively fast (or slow) with regards to the characteristics time, i.e. $\mathrm{f}=0.01$ and $1 \mathrm{~Hz}$, insignificant (less pronounced) time-dependent effect is shown, indicated by narrow ellipsoidal shapes. This is because under a relatively fast loading, the material does not have enough time to experience delay changes at the microstructures; while under a relatively slow loading these delay changes at the microstructures are (nearly) complete. The creep functions in the electro-mechanical coupling results in a smaller slope of the electric field-strain curve when a lower frequency is considered. Figure 3.4 depicts the linear response at different magnitudes of electric fields, which show a perfect elliptical shape when saturated condition is reached.

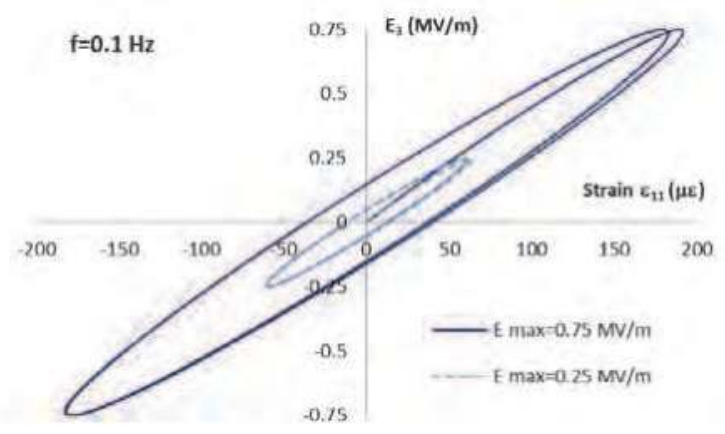

Fig. 3.4. The effect of the amplitude of the electric field on the linear hysteretic response $(\mathrm{f}=0.1 \mathrm{~Hz})$

We use a single integral model with nonlinear integrands to illustrate the hysteretic response of a polarized PZT. The following nonlinear functions of the integral model in Eq. (2.13) are chosen for the electro-mechanical coupling $E_{3}-\varepsilon_{11}$ :

$$
\varepsilon_{11}(t)=-200\left(1+\alpha\left|E_{3}\right|\right) E_{3}-100\left(1+\beta\left|E_{3}\right|\right) E_{3}\left(1 .-e^{-t / 2}\right) \quad\left(10^{-12} \mathrm{C} / N\right)
$$

Figure 3.5 illustrates the effect of different nonlinear parameters on the hysteretic electromechanical response. We use nonlinear functions that vary linearly with electric field; it is also possible to pick different functions. We assume that the electro-mechanical coupling properties increase with increasing the magnitude of the electric field and we also assume

${ }^{5}$ It is noted that we measure these time-dependent changes with respect to the laboratory (experimental) time at the macroscopic level. 
that the material relaxes faster with increase in the magnitude of the electric field. The following material constants are used in the numerical simulations: $\alpha=\beta=0.5 \mathrm{~m} / \mathrm{MV}$ and the characteristics time varies with the magnitude of electric field as: $\left(1+\gamma\left|E_{3}\right|\right) 2 ; \gamma=-0.75$. In this case, we are interested in the response of piezoceramics below the coercive electric field such that the piezoceramics does not experience polarization switching. We also assume that applying electric fields along and opposite to the poling axis cause similar changes in the corresponding strains ${ }^{6}$. The nonlinear parameters show distortion in the hysteretic response from an ellipsoidal shape. As in the linear case, we also show the effect of the amplitude of the electric field on the nonlinear hysteretic response. All of the above nonlinear material parameters are incorporated in the numerical simulations. Figure 3.6 shows the hysteretic response obtained from the nonlinear single integral model. The deviation from the ellipsoidal shape is more pronounced for the hysteretic response under the highest magnitude of the electric field, which is expected. Under relatively small amplitude of the electric field, the hysteretic response shows almost a perfect ellipse as the nonlinearity is less pronounced.

In the third case study, we apply a constant stress input together with a sinusoidal electric field input:

$$
\sigma_{33}(t)=-20 H(t) M P a \quad E_{3}(t)= \pm 0.75 \sin \omega t M V / m
$$

where $H(t)$ is the Heaviside unit step input. The following time-dependent compliance and linear electro-mechanical coupling constant are considered 7 :

$$
\begin{aligned}
& S_{3333}(t)=0.0122\left(1.5-0.5 e^{-t / 50}\right) G P a^{-1} \\
& d_{333}(t)=\left[380+150\left(1 .-e^{-t / 5}\right)\right] \cdot 10^{-12} \mathrm{C} / N(m / V)
\end{aligned}
$$

The above compliance corresponds to the elastic (instantaneous) modulus $E_{33}$ of $82 \mathrm{GPa}$. In the linear model the strain output due to the applied compressive stress can be superposed with the strain output due to the applied electric field. Under a relatively high compressive stress applied along the poling axis depoling of the PZT could occur, leading to nonlinear response. The scope of this manuscript is not on simulating a polarization reversal behavior and we assume that the superposition condition is applicable for the time-dependent strain outputs due to stress and electric field inputs. We allow the polarized PZT to experience creep when it is subjected to a stress. The creep response is described by the compliance in Eq. (3.4). A sinusoidal electric field with amplitude of $0.75 \mathrm{MV} / \mathrm{m}$ and frequency of $0.1 \mathrm{~Hz}$ is applied. Two cases regarding the history of the electric field input are considered: The first case starts with applying the electric field in the opposite direction to the poling axis, $E_{3}\left(0^{+}\right)<0.0$. The second case starts with the electric field input in the direction of the

\footnotetext{
${ }^{6}$ It is noted that the corresponding strain response in a polarized ferroelectric ceramics when the electric field is applied along the poling axis need not be the same as the strain output when the electric field is applied opposite to the poling axis. In most cases they are not the same, especially under a relative high magnitude of electric field as the process of polarization switching might occur even before it reaches the coercive electric field.

7 The PZT is modeled as a viscoelastic solid with regards to its mechanical response. The creep deformation in a viscoelastic solid will reach an asymptotic value at steady state (saturated condition).
} 
poling axis, $E_{3}\left(0^{+}\right)>0.0$. When an electric field is applied opposite to the current poling axis, the PZT experiences contraction in the poling direction, indicated by a compressive strain. When the polarized PZT is subjected to an electric field in the poling direction, it experiences elongation in that direction.
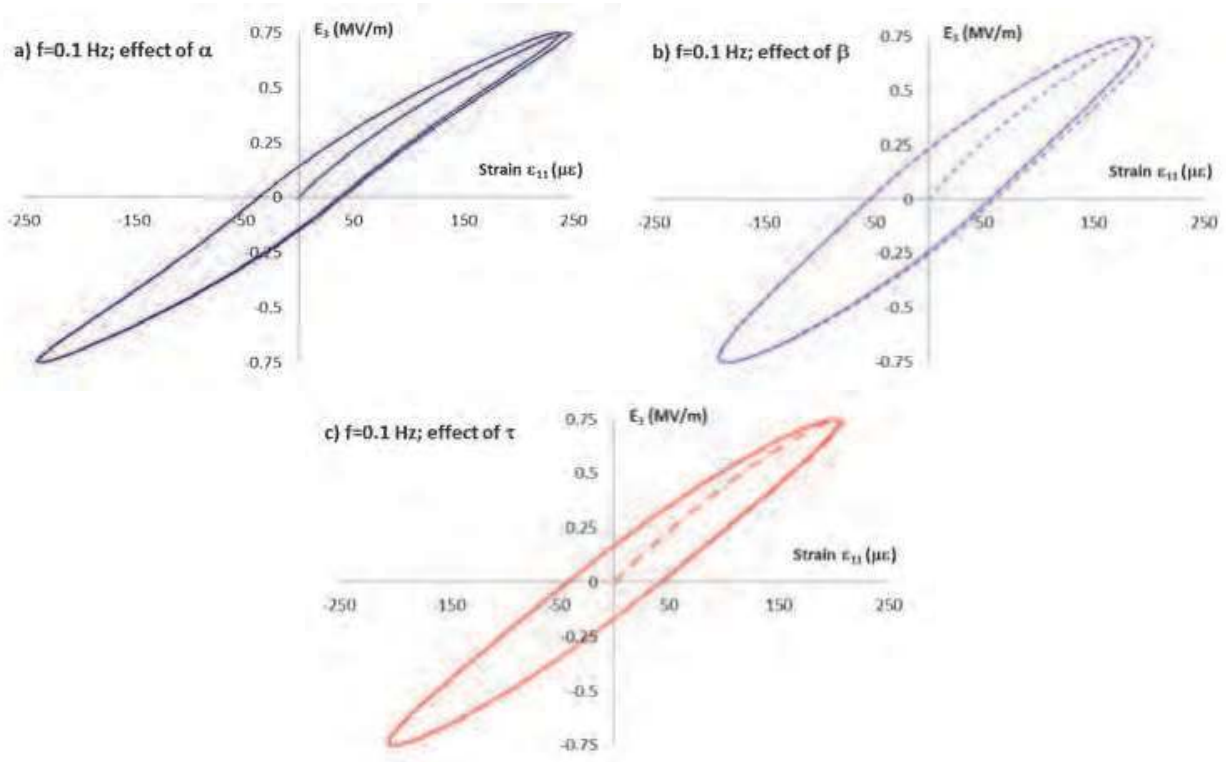

Fig. 3.5. The effect of nonlinear parameters on the hysteretic response

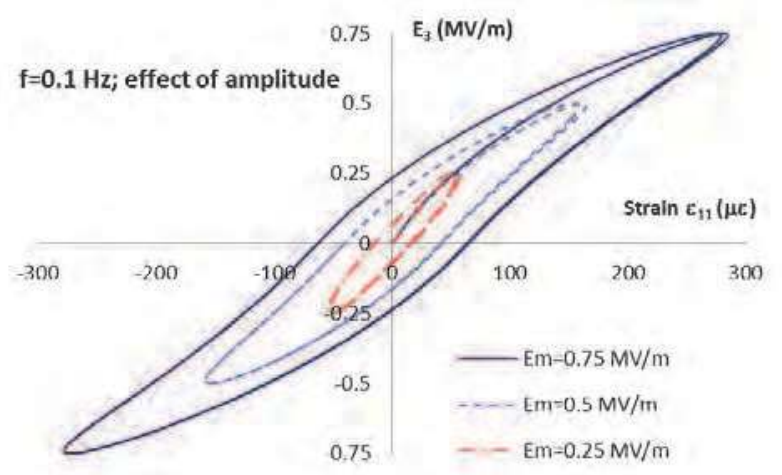

Fig. 3.6. The effect of the amplitude of the electric field on the nonlinear hysteretic response $(\mathrm{f}=0.1 \mathrm{~Hz})$

We examine the effect of the electric field input history on the corresponding strain output when the PZT undergoes creep deformation. Figure 3.7 illustrates the hysteretic response under the input field variables in Eq. 3.3. As expected, the creep deformation in the PZT due 
to the compressive stress continuously shifts the hysteretic response to the left of the strain axis (higher values of the compressive strains) until steady state is reached for the creep deformation. At steady state, the hysteretic response should form an ellipsoidal shape. It is also seen that different hysteretic response is shown under the two histories of electric fields discussed above. When the electric field is first applied opposite to the poling axis, the first loading cycle forms a nearly elliptical hysteretic response. This is not the case when the electric field is first applied in the poling direction (Fig. 3.7b). The hysteretic response under a frequency of $1 \mathrm{~Hz}$ is also illustrated in Figs. 3.7c and d, which show an insignificant timedependent effect. This is due to the fact that the rate of loading under $\mathrm{f}=1 \mathrm{~Hz}$ is much faster as compared to the creep and time-dependent response of the material. It is also seen that under frequency $1 \mathrm{~Hz}$, the strain- and electric field response is almost linear. Thus, under such condition it is possible to characterize the linear piezoelectric constants of materials, i.e. $d_{311}, d_{322}, d_{333}, d_{113}, d_{223}$ from the electric field-strain curves. At this frequency of $1 \mathrm{~Hz}$, the slope in the strain-electric field curves (Figs. 3.7c and d) remains almost unaltered with the history of the applied electric field. This study can be useful for designing an experiment and interpreting data in order to characterize the piezoelectric properties of a piezoelectric ceramics.

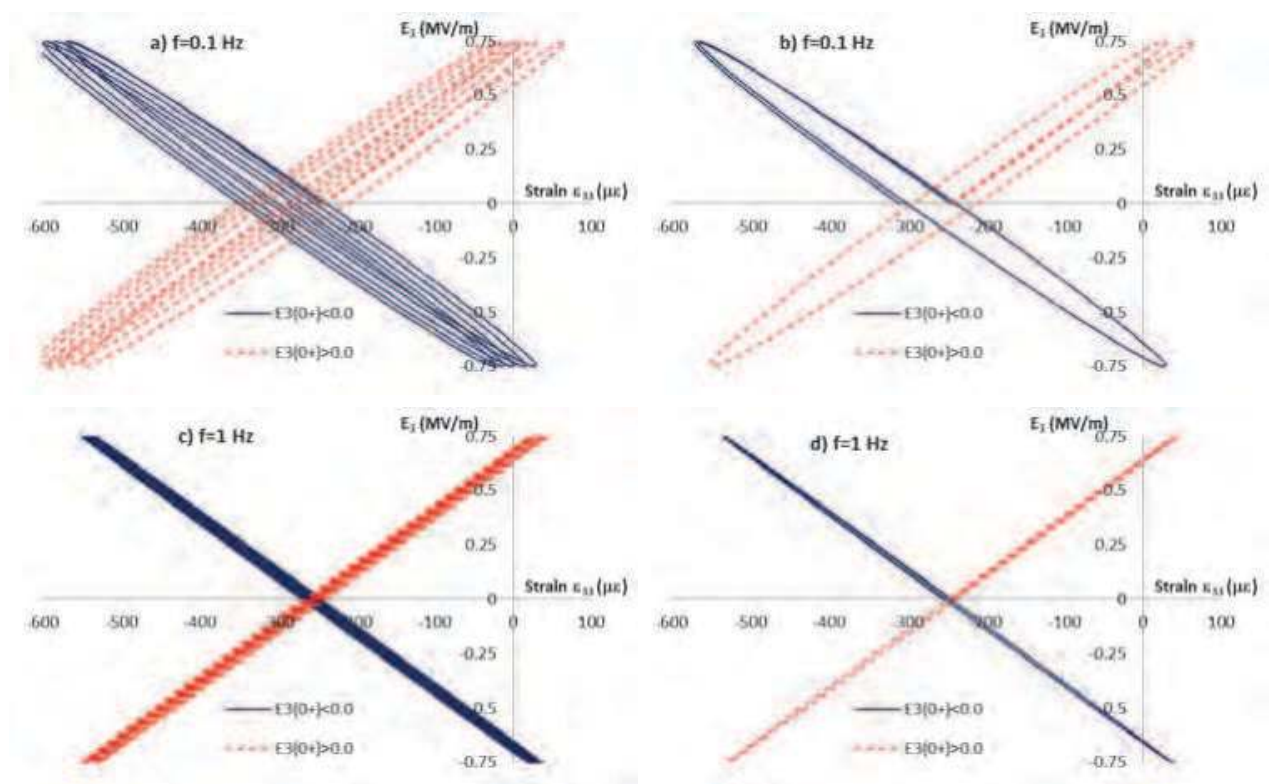

Fig. 3.7. The corresponding hysteretic response under coupled mechanical and electric field inputs

\subsection{Multiple integral model}

This section presents a multiple integral model to simulate hysteretic response of a piezoelectric ceramics subject to a sinusoidal electric field. We consider up to the third order kernel function and we examine the effect of these kernel functions on the overall nonlinear hysteretic curve. The following material parameters are used for the simulation: 


$$
\begin{aligned}
& A_{0}=200 \cdot 10^{-12} \mathrm{~m} / \mathrm{V} ; A_{1}=100 \cdot 10^{-12} \mathrm{~m} / \mathrm{V} \\
& \tau_{1}=2 \mathrm{sec} \\
& B_{0}=B_{1}=B_{2}=20 \cdot 10^{-18} \mathrm{~m}^{2} / \mathrm{V}^{2} \\
& \lambda_{1}=2 \mathrm{sec} ; \lambda_{2}=5 \mathrm{sec} \\
& C_{0}=C_{1}=C_{2}=50 \cdot 10^{-24} \mathrm{~m}^{3} / \mathrm{V}^{3} \\
& \eta_{1}=2 \mathrm{sec} ; \eta_{2}=5 \mathrm{sec}
\end{aligned}
$$

When only the first and third kernel functions are considered, the nonlinear hysteretic response at steady state under positive and negative electric fields is identical as shown by an anti-symmetric hysteretic curve in Fig. 3.8a. The hysteretic response under the amplitude of electric field of $0.25 \mathrm{MV} / \mathrm{m}$ shows nearly linear response. Including the second order kernel function allows for different response under positive and negative electric fields as seen in Fig. 3.8b. At low amplitude of applied electric field, nearly linear response is shown; however this hysteretic response does not show an anti-symmetric shape with respect to the strain and electric field axes. The contribution of each order of the kernel function depends on the material parameters. For example the material parameters in Eq. (3.5) yield to more pronounced contribution of the first order kernel function; while the contributions of the second and third order kernel functions are comparable.
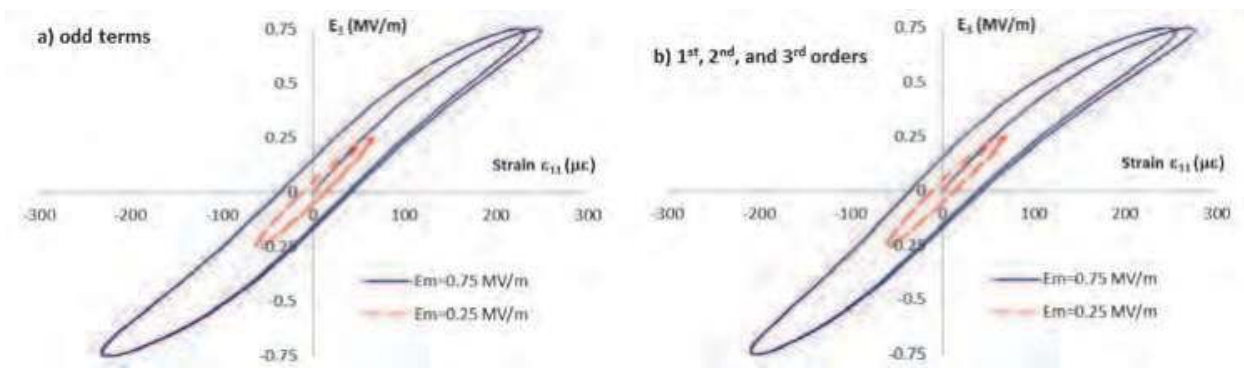

Fig. 3.8. The effect of the higher order terms on the hysteretic response $(\mathrm{f}=0.1 \mathrm{~Hz})$

Intuitively, the corresponding strain response of a piezoelectric ceramics when an electric field is applied in the poling direction (positive electric field) need not be the same as when an electric field is applied opposite to the poling direction (negative electric field), especially for nonlinear response due to high electric fields. Depoling could occur in the piezoelectric ceramics when a negative electric field with a magnitude greater than the coercive electric field is considered. Thus, to incorporate the possibility of the depoling process, the even order kernel functions can be incorporated in the multiple time-integral model. In order to numerically simulate the depolarization in the piezoelectric ceramics we apply a sinusoidal electric field input with amplitude of $1.5 \mathrm{MV} / \mathrm{m}$. We consider the first and second order kernel functions and use the following material parameters so that the contributions of the first and second order kernel functions on the strain response are comparable:

$$
\begin{aligned}
& A_{0}=200 \cdot 10^{-12} \mathrm{~m} / \mathrm{V} ; A_{1}=100 \cdot 10^{-12} \mathrm{~m} / \mathrm{V} \\
& \tau_{1}=2 \mathrm{sec} \\
& B_{0}=B_{1}=B_{2}=100 \cdot 10^{-18} \mathrm{~m}^{2} / \mathrm{V}^{2} \\
& \lambda_{1}=2 \mathrm{sec} ; \lambda_{2}=5 \mathrm{sec}
\end{aligned}
$$


Figure 3.9 illustrates the corresponding strain response from the multiple integral model having the first and second kernel functions. The response shows an un-symmetric butterfly-like shape. The un-symmetric butterfly-like strain-electric field response is expected for polarized ferroelectric materials undergoing high amplitude of sinusoidal electric field input. The nonlinear response due to the positive electric field is caused by different microstructural changes than the microstructural changes due to polarization switching under a negative electric field.

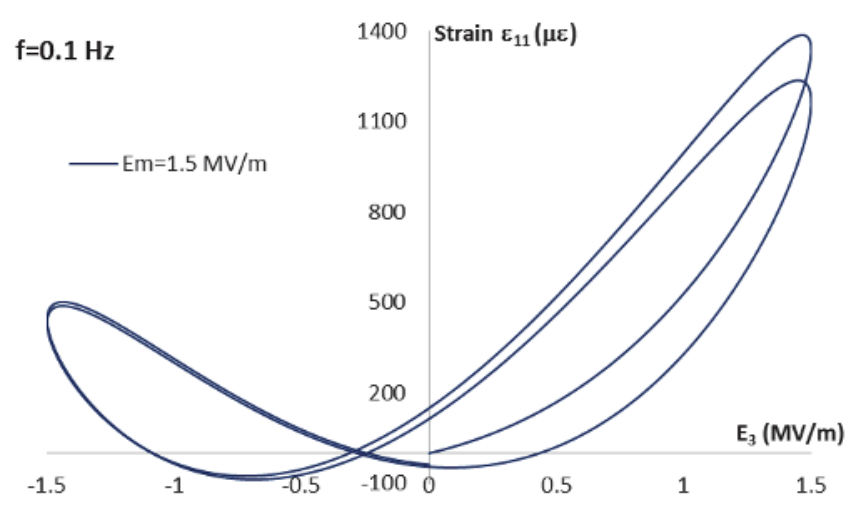

Fig. 3.9. The butterfly-like shape of the electro-mechanical coupling response

\section{Analyses of piezoelectric beam bending actuators}

Stack actuators have been used in several applications that involve displacement controlling, such as fuel injection valves and optical positioning (see Ballas 2007 for a detailed discussion). They comprise of layers of polarized piezoelectric ceramics arranged in a certain way with regards to the poling axis of an individual piezoceramic layer in order to produce a desire deformation. In conventional bending actuators, a single layer piezoceramic requires a typical of operating voltage of $200 \mathrm{~V}$ or more. By forming a multilayer piezoceramic actuator, it is possible to reduce the operating voltage to less than $50 \mathrm{~V}$. In this section, we examine the effect of time-dependent electro-mechanical properties of the piezoelectric ceramics on the bending deflections of an actuator comprising of two piezoelectric layers, known as a bimorph system.

Consider a two dimensional bimorph beam consisting of two layers of polarized piezoelectric ceramics and an elastic layer, as shown in Fig. 4.1. In order to produce a bending deflection in the beam, the two piezoelectric layers should undergo opposite tensile and compressive strains. This can be achieved by stacking the two piezoelectric layers with the poling axis in the same direction and applying a voltage that produces opposite electric fields in the two layers or by placing the two piezoelectric layers with poling axis in the opposite direction and applying a voltage that produces electric fields in the same direction. The beam is fixed at one end and the other end is left free; the top and bottom surfaces are under a traction free condition. A potential is applied at the top and bottom surfaces of the beam and the corresponding displacement is monitored. We prescribe the following boundary conditions to the bimorph beam: 


$$
\begin{aligned}
& u_{1}\left(0, x_{2}, t\right)=u_{2}\left(0, x_{2}, t\right)=\frac{\partial u_{2}}{\partial x_{1}}\left(0, x_{2}, t\right)=0 \quad-\frac{h}{2} \leq x_{2} \leq \frac{h}{2}, t \geq 0 \\
& \sigma_{22}\left(x_{1}, \frac{h}{2}, t\right)=\sigma_{22}\left(x_{1},-\frac{h}{2}, t\right)=0 \quad 0 \leq x_{1} \leq L, t \geq 0 \\
& \sigma_{12}\left(L, x_{2}, t\right)=0 \quad-\frac{h}{2} \leq x_{2} \leq \frac{h}{2}, t \geq 0 ; \sigma_{12}\left(x_{1}, \frac{h}{2}, t\right)=\sigma_{12}\left(x_{1},-\frac{h}{2}, t\right)=0 \quad 0 \leq x_{1} \leq L, t \geq 0 \\
& \varphi\left(x_{1}, \frac{h s}{2}, t\right)=\varphi\left(x_{1},-\frac{h s}{2}, t\right)=0 \quad 0 \leq x_{1} \leq L, t \geq 0 \\
& \varphi\left(x_{1}, \frac{h}{2}, t\right)=\varphi\left(x_{1},-\frac{h}{2}, t\right)=V_{h}(t) \quad 0 \leq x_{1} \leq L, t \geq 0
\end{aligned}
$$

where $u_{1}$ and $u_{2}$ are the displacements in the $x_{1}$ and $x_{2}$ directions, respectively. The bonding between the different layers in the bimorph beam is assumed perfect; thus the traction and displacement continuity conditions are imposed at the interface layers. The beam has a length $L$ of $100 \mathrm{~mm}$, width $b$ of $1 \mathrm{~mm}$ and the thickness of each piezoelectric layer is $1 \mathrm{~mm}$. Let us consider a bimorph beam without an elastic layer placed in between these piezoelectric layers. We assume that the beam is relatively slender so that it is sensible to adopt Euler-Bernoulli's beam theory in finding the corresponding displacement of the bimorph beam; the calculated displacements are at the neutral axis of the beam and we shall eliminate the dependence of the displacements on the $x_{2}$ axis, $u_{1}\left(x_{1}, t\right)$ and $u_{2}\left(x_{1}, t\right)$. The kinematics concerning the deformations of the Euler-Bernoulli beam, with the displacements measured at the neutral axis of the beam is:

$$
\varepsilon_{11}\left(x_{1}, x_{2}, t\right)=\frac{\partial u_{1}}{\partial x_{1}}\left(x_{1}, t\right)-x_{2} \frac{\partial^{2} u_{2}}{\partial x_{1}^{2}}\left(x_{1}, t\right)
$$

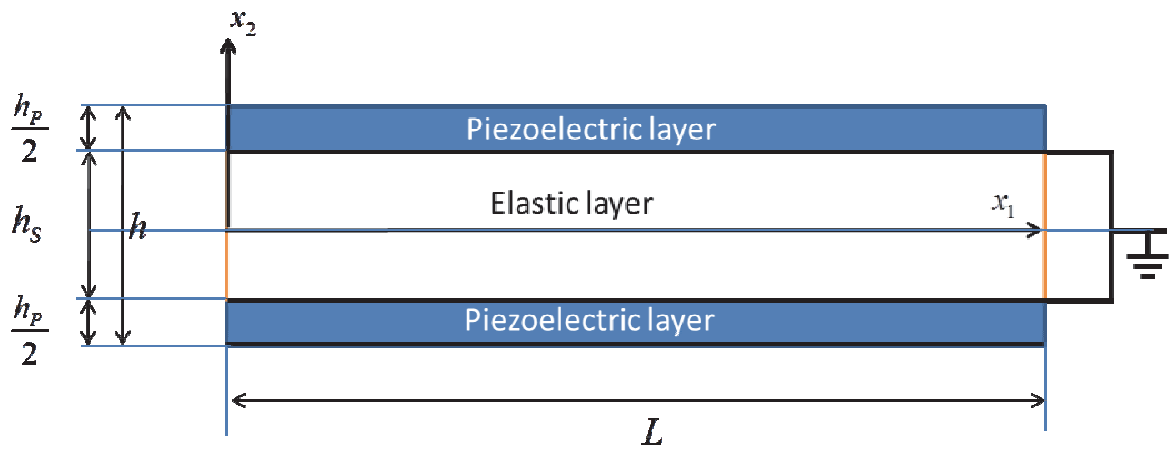

Fig. 4.1. A bimorph beam

Since we only prescribe a uniform voltage on the top and bottom surfaces of the beam, the problem reduces to a pure bending problems: the internal bending moment depends only on

\footnotetext{
${ }^{8}$ We shall only consider the longitudinal stress- and strain and the transverse displacement measured at the neutral axis of the beam.
} 
time, $M_{3}(t)=M(t)$ and the longitudinal stress is independent on the $x_{1}, \sigma_{11}\left(x_{2}, t\right)$. At each time $t$, the following equilibrium conditions must be satisfied:

$$
\begin{aligned}
& \int_{A} \sigma_{11}\left(x_{2}, t\right) d A=0 \\
& M(t)=-\int_{A} x_{2} \sigma_{11}\left(x_{2}, t\right) d A
\end{aligned}
$$

As a consequence, the first term of the axial strain in Eq. (4.2) is zero and the curvature of the beam depends only on time. The constitutive relations for the piezoelectric layers are:

$$
\begin{aligned}
& \sigma_{11}\left(x_{2}, t\right)=\int_{0^{-}}^{t} \frac{\partial C_{1111}}{\partial s}\left(x_{2}, t-s\right) \varepsilon_{11}\left(x_{2}, s\right) d s-\int_{0^{-}}^{t} \frac{\partial e_{211}}{\partial s}\left(x_{2}, t-s\right) E_{2}\left(x_{2}, s\right) d s \\
& D_{2}\left(x_{2}, t\right)=\int_{0^{-}}^{t} \frac{\partial e_{211}}{\partial s}\left(x_{2}, t-s\right) \varepsilon_{11}\left(x_{2}, s\right) d s+\int_{0^{-}}^{t} \frac{\partial \kappa_{22}}{\partial s}\left(x_{2}, t-s\right) E_{2}\left(x_{2}, s\right) d s
\end{aligned}
$$

where the electric field at the piezoelectric layer with the thickness $h_{p} / 2$ is assumed uniform $E_{2}\left(x_{2}, t\right)=-\frac{V_{h}(t)}{h_{p} / 2}$ for $0 \leq x_{2} \leq \frac{h_{p}}{2}$ and $E_{2}\left(x_{2}, t\right)=\frac{V_{h}(t)}{h_{p} / 2}$ for $-\frac{h_{p}}{2} \leq x_{2} \leq 0$. The poling axes of the two piezoelectric layers are in the same direction. The axial stress becomes $\left(h_{s}=0\right)$ :

$$
\sigma_{11}\left(x_{2}, t\right)=-\left\{\begin{array}{lc}
-\frac{2}{h_{p}} \int_{0^{-}}^{t} \frac{\partial e_{211}}{\partial s}(t-s) V_{h}(s) d s & 0 \leq x_{2} \leq \frac{h_{p}}{2} \\
\frac{2}{h_{p}} \int_{0^{-}}^{t} \frac{\partial e_{211}}{\partial s}(t-s) V_{h}(s) d s & -\frac{h_{p}}{2} \leq x_{2} \leq 0
\end{array}\right.
$$

Substituting the stress in Eq. (4.5) to the internal bending moment in Eq. (4.3) yields to:

$$
M(t)=b \frac{h_{p}}{2} \int_{0^{-}}^{t} \frac{\partial e_{211}}{\partial s}(t-s) V_{h}(s) d s
$$

Finally, the equation that governs the bending of the bimorph beam (pure bending condition) subject to a time varying electric potential is:

$$
\frac{\partial^{2} u_{2}}{\partial x_{1}^{2}}=\frac{1}{I} \int_{0^{-}}^{t} \frac{\partial S_{1111}}{\partial s}(t-s) M(s) d s=\frac{b}{I} \frac{h_{p}}{2} \int_{0^{-}}^{t} \frac{\partial S_{1111}}{\partial s}(t-s) \int_{0^{-}}^{s} \frac{\partial e_{211}}{\partial s}(s-\zeta) V_{h}(\zeta) d \zeta d s=\Phi(t)
$$

where $I$ is the second moment of an area w.r.t. the neutral axis of the beam. Integrating Eq. (4.7) with respect to the $x_{1}$ axis and using BCs in Eq. 4.1, the deflection of the beam is:

$$
u_{2}\left(x_{1}, t\right)=\frac{1}{2} \Phi(t) x_{1}^{2}
$$

The following time-dependent properties of PZT-5A are used for the bending analyses of stack actuators: 


$$
\begin{aligned}
& C_{1111}(t)=\left[90+30 e^{-t / 50}\right] \mathrm{GPa} \\
& e_{211}(t)=-\left[5.35+1.34 e^{-t / 5}\right] \mathrm{C} / \mathrm{m}^{2}
\end{aligned}
$$

A sinusoidal input of an electric potential with various frequencies are applied. Figure 4.2 illustrates hysteresis response of the bending of the bimorph beam. The displacements are measured at the free end $\left(x_{1}=100 \mathrm{~mm}\right)$. As discussed in Section 3.1, when the rate of loading is comparable to the characteristics time, the effect of time-dependent material properties on the hysteretic response becomes significant, as shown by the response with frequencies of $0.05 \mathrm{~Hz}$ and $0.1 \mathrm{~Hz}$. When the rate of loading is relatively fast (or slow) with regards to the characteristics time, i.e. $\mathrm{f}=0.01 \mathrm{~Hz}$ and $1 \mathrm{~Hz}$, insignificant (less pronounced) time-dependent effect is shown, indicated by narrow ellipsoidal shapes.
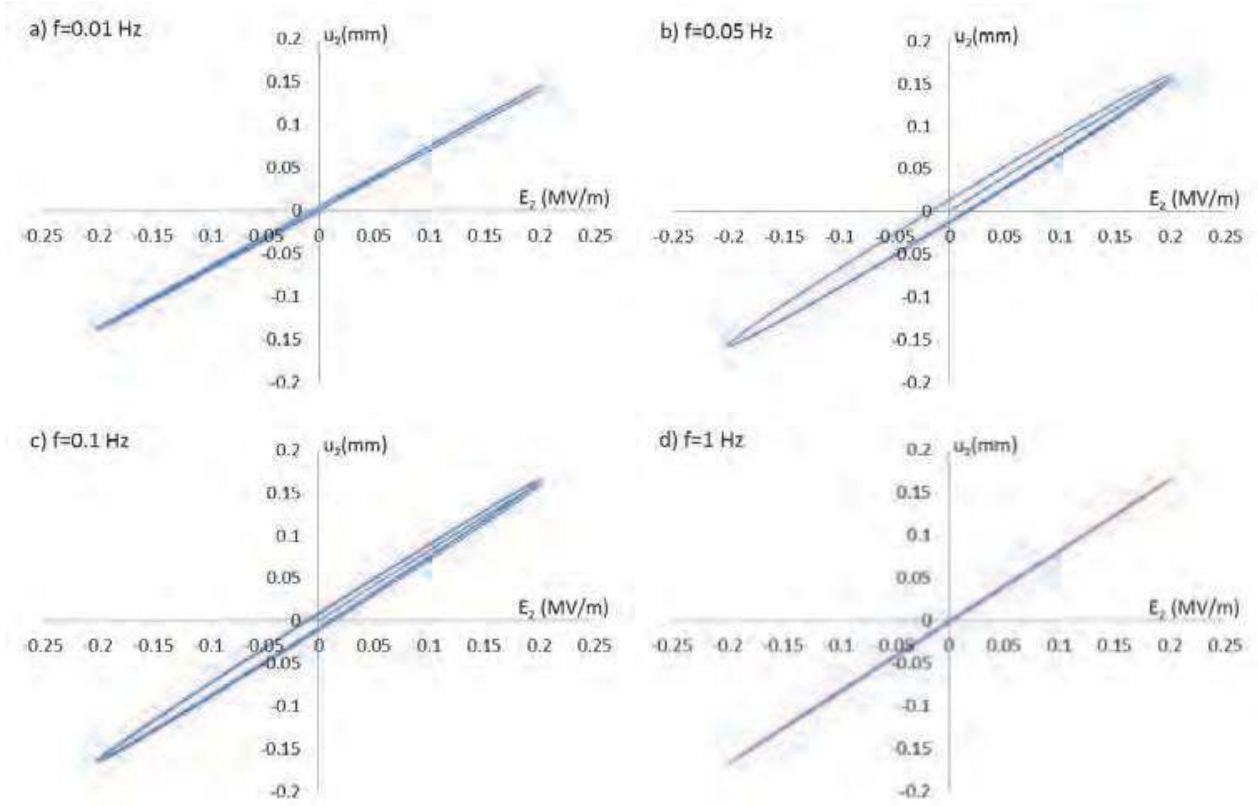

Fig. 4.2. The effect of input frequencies on the tip displacements of the bimorph beam

\section{Conclusions}

We have studied the nonlinear and time-dependent electro-mechanical hysteretic response of polarized ferroelectric ceramics. The time-dependent electro-mechanical response is described by nonlinear single integral and multiple integral models. We first examine the effect of frequency (loading rate) on the overall hysteretic response of a linear timedependent electro-mechanical response. The strain-electric field response shows a nonlinear relation when the time-dependent effect is prominent which should not be confused with the nonlinearity due to the magnitude of electric fields. We also study the effect of the magnitude of electric fields on the overall hysteretic response using both nonlinear single integral and multiple integral models. As expected, the nonlinearity due to the electric field 
results in a distortion of the ellipsoidal hysteretic curve. We have extended the timedependent constitutive model for analyzing bending in a stack actuator due to an input electric potential at various frequencies. The presented study will be useful when designing an experiment and interpreting data that a nonlinear electro-mechanical response exhibits. This study is also useful in choosing a proper nonlinear time-dependent constitutive model for piezoelectric ceramics.

\section{Acknowledgment}

This research is sponsored by the Air Force Office of Scientific Research (AFOSR) under grant FA 9550-10-1-0002.

\section{References}

[1] Ballas, RG (2007) Piezoelectric Multilayer Beam Bending Actuators, Springer-Verlag Berlin

[2] Bassiouny, E., Ghaleb, AF, and Maugin GA (1988a), "Thermodynamical Formulation for Coupled Electromechanical Hysteresis Effects-I. Basic Equations," Int. J. Engrg Sci., 26, pp. 1279-1295

[3] Bassiouny, E., Ghaleb, AF, and Maugin GA (1988b), “Thermodynamical Formulation for Coupled Electromechanical Hysteresis Effects-I. Poling of Ceramics," Int. J. Engrg Sci., 26, pp. 975-987

[4] Ben Atitallah, H, Ounaies, Z, and Muliana A, "Temperature and Time Effects in the Electro-mechanical Coupling Behavior of Active Fiber Composites", 16th US National Congress on Theoretical and Applied Mechanics, June 27 - July 2, 2010, State College, Pennsylvania, USA

[5] Cao, H. and Evans, A.G. (1993), "Nonlinear Deformation of Ferroelectric Ceramics" J. Amer. Ceramic Soc., 76, pp. 890-896

[6] Chan, K.H. and Hagood, N.W. (1994), "Modeling of Nonlinear Piezoceramics for Structural Actuation," Proc. of SPIE's Symp. on Smart Structures and Materials, 2190, pp. 194-205

[7] Chen, W. and Lynch, C.S. (1998), “A Micro-electro-mechanical Model for Polarization Switching of Ferroelectric Materials," Acta Materialia, 46, pp. 5303-5311

[8] Chen, X. (2009), "Nonlinear Electro-thermo-viscoelasticity," Acta Mechanica, in press

[9] Crawley, E.F. and Anderson, E.H. (1990), “Detailed Models of Piezoceramic Actuation of Beams" J. Intell. Mater. Syst. Struct., 1, pp. 4-25

[10] Fett, T. and Thun, G. (1998), "Determination of Room-temperature Tensile Creep of PZT," J. Materials Science Letter, 17, pp. 1929-1931

[11] Green, AE and Rivlin, RS (1957), "The Mechanics of Nonlinear Materials with Memory, Part I," Archive for Rational Mechanics and Analysis, 1, pp. 1

[12] Fang, D. and Sang Y. (2009), "The polarization properties in ferroelectric nanofilms investigated by molecular dynamics simulation, "Journal of Computational and Theoretical Nanoscience, 6, pp. 142-147

[13] Findley, W. and Lai., J (1967), “A Modified Superposition Principle Applied to Creep of Nonlinear Viscoelastic Material Under Abrupt Changes in State of Combined Stress" Trans. Soc. Rheol., 11, pp. 361 
[14] Findley, WN, Lai, JS, Onaran, K (1976), “Creep and Relaxation of Nonlinear Viscoelastic Materials," Dover Publication, New York

[15] Fung, Y.C (1981) Biomechanics: Mechanical Properties of Living Tissues, Springer, New York

[16] Hall, D.A. (2001), “Review Nonlinearity in Piezoelectric Ceramics," J. Materials Sci, 36, pp. $4575-4601$

[17] Huang L and Tiersten HF (1998), "Electroelastic equations describing slow hysteresis in polarized ferroelectric ceramic plates," J. App. Physics, 83, pp. 6126-6139

[18] Huang L and Tiersten HF (1998), "An Analytical Description of Slow Hysteresis in Polarized Ferroelectric Ceramic Actuators," J. Intel. Mater. Syst. and Struct., 9, pp. 417-426

[19] Kamlah, M. and Tsakmakis, C (1999), "Phenomenological Modeling of the Nonlinear Electro-mechanical Coupling in Ferroelectrics," Int. J. Solids and Structures, 36, pp. 669-695

[20] Landis, C. (2002), "A phenomenological multiaxial constitutive law for switching in polycrystalline ferrorlectric ceramics," J. Mech. Phys. Solids, vol. 50, pp. 127-152

[21] Li J. and Weng GJ (2001), "A Micromechanics-Based Hysteresis Model for Ferroelectric Ceramics," J. Intel. Material Systems and Structures, 12, pp. 79-91

[22] Lines, M.E. and Glass, A. E. (2009) Principles and Applications of Ferroelectrics and Related Materials, Oxford University Press, New York

[23] Massalas, C.V., Foutsitzi, G., Kalpakidis, V.K. (1994), “Thermoelectroelasticity Theory for Materials with Memory," Int. J. Engng. Sci., 7, pp. 1075-1084

[24] Pipkin, A.C. and Rogers, T.G. (1968), “A nonlinear integral representation for viscoelastic behavior," J. Mech. Phys. Solids, 16, pp. 69-72

[25] Rajagopal, K. R. and Wineman, A. S. (2010), "Application to Viscoelastic Clock Models in Biomechanics," Acta Mechanica, 213, pp. 255-266

[26] Schaeufele, A. and Haerdtl, K.H. (1996), "Ferroelastic Properties of Lead Zirconate Titanate Ceramics" J. Amer. Ceramic Soc., 79, pp. 2637-2640

[27] Schapery, R. A. (1969), "On the Characterization of Nonlinear Viscoelastic Materials," Polymer Engineering and Science, Vol. 9, No. 4, pp. 295-310

[28] Smith, R.C., Seelecke, S., Ounaies, Z., and Smith, J. (2003), “A Free Energy Model for Hysteresis in Ferroelectric Materials," J. Intel. Mater. Syst. and Structures, 14, pp. 719-739

[29] Smith, R.C., Seelecke, S., Dapino, M., Ounaies, Z. (2006), "A unified framework for modeling hysteresis in ferroic materials," J. Mech. Phys. Solids, 54, pp. 46-85

[30] Smith, RC (2005), Smart Material Systems: Model Development. Philadelphia, PA: SIAM

[31] Tiersten, H.F. (1971), “On the Nonlinear Equations of Thermo-electroelasticity," Int. J. Engr. Sci, Vol. 9, pp. 587-604

[32] Tiersten, H.F. (1993), "Electroelastic Equations for Electroded Thin Plates Subject to Large Driving Voltages" J. Applied Physics, 74, pp. 3389-3393

[33] Uludogan M, Guarin, DP, Gomez, ZE, Cagin, T, and Goddard III, WA (2008), “DFT studies on ferroelectric ceramics and their alloys: BaTiO3, PbTiO3, SrTiO3, AgNbO3, AgTaO3, PbxBa1-xTiO3 and SrxBa 1-xTiO3", Computer Modeling in Engineering and Sciences, 24, pp. 215-238 
[34] Zhou, D. and Kamlah, M. (2006), “Room-temperature Creep of Soft PZT under Static Electrical and Compressive Stress Loading," Acta Materialia, 54, pp. 1389-1396 


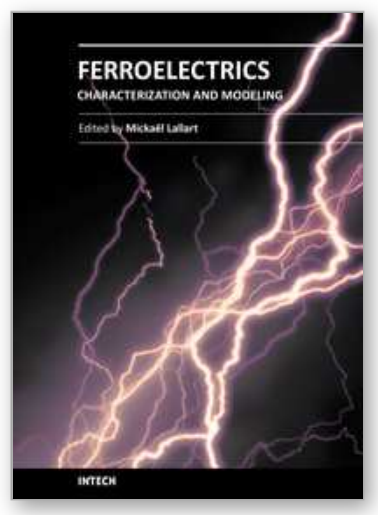

\author{
Ferroelectrics - Characterization and Modeling \\ Edited by Dr. MickaÃ«l Lallart
}

ISBN 978-953-307-455-9

Hard cover, 586 pages

Publisher InTech

Published online 23, August, 2011

Published in print edition August, 2011

Ferroelectric materials have been and still are widely used in many applications, that have moved from sonar towards breakthrough technologies such as memories or optical devices. This book is a part of a four volume collection (covering material aspects, physical effects, characterization and modeling, and applications) and focuses on the characterization of ferroelectric materials, including structural, electrical and multiphysic aspects, as well as innovative techniques for modeling and predicting the performance of these devices using phenomenological approaches and nonlinear methods. Hence, the aim of this book is to provide an up-to-date review of recent scientific findings and recent advances in the field of ferroelectric system characterization and modeling, allowing a deep understanding of ferroelectricity.

\title{
How to reference
}

In order to correctly reference this scholarly work, feel free to copy and paste the following:

Amir Sohrabi and Anastasia Muliana (2011). Nonlinear Hysteretic Response of Piezoelectric Ceramics, Ferroelectrics - Characterization and Modeling, Dr. MickaÃ«I Lallart (Ed.), ISBN: 978-953-307-455-9, InTech, Available from: http://www.intechopen.com/books/ferroelectrics-characterization-and-modeling/nonlinearhysteretic-response-of-piezoelectric-ceramics

\section{INTECH}

open science | open minds

\section{InTech Europe}

University Campus STeP Ri

Slavka Krautzeka 83/A

51000 Rijeka, Croatia

Phone: +385 (51) 770447

Fax: +385 (51) 686166

www.intechopen.com

\section{InTech China}

Unit 405, Office Block, Hotel Equatorial Shanghai

No.65, Yan An Road (West), Shanghai, 200040, China 中国上海市延安西路65号上海国际贵都大饭店办公楼 405 单元

Phone: +86-21-62489820

Fax: $+86-21-62489821$ 
(C) 2011 The Author(s). Licensee IntechOpen. This chapter is distributed under the terms of the Creative Commons Attribution-NonCommercialShareAlike-3.0 License, which permits use, distribution and reproduction for non-commercial purposes, provided the original is properly cited and derivative works building on this content are distributed under the same license. 